\title{
Diferentes Abordagens de Computação Afetiva em Sistemas Multiagentes e Sistemas Tutores Inteligentes
}

Edilson Pontarolo Magda Bercht Rosa Maria Vicari

\section{Different Affective Computing Approachs in Multiagent and Intelligent Tutoring Systems}


Resumo: Os resultados recentemente obtidos nas neurociĺncias e ciĺncias cognitivas apontam para novas explicaÁıes sobre o papel da emoÁ,,o na aprendizagem e outros processos cognitivos. Esse entendimento influenciou, sobretudo a partir da dÈcada de 1990, a pesquisa e o desenvolvimento de sistemas e ambientes computacionais de aprendizagem. Este artigo apresenta algumas abordagens de computaÁ, „o afetiva tal como vím sendo adotadas por diferentes grupos de pesquisa, no formalismo simbÛlico de linguagens e racioclnio para o desenvolvimento de sistemas multiagentes e/ou de sistemas tutores inteligentes.

Palavras-chave: ComputaÁ,o afetiva. Sistemas multiagentes. Sistemas tutores inteligentes.

Abstract: The recently obtained results from neuro and cognitive sciences point to new explanations about the emotion role in learning and other cognitive processes. This understanding influenced, overall starting from the 1990 decade, the research on computer systems and learning environments. This paper presents some affective computing approachs as adopted by different research groups, on the symbolic formalism of languages and reasoning in multiagent and/or intelligent tutoring systems development.

Keywords: Affective computing. Multiagent systems. Intelligent tutoring systems.

PONTAROLO, Edilson; BERCHT, Magda; VICARI, Rosa Maria. Diferentes Abordagens de ComputaÁ,,o Afetiva em Sistemas Multiagentes e Sistemas Tutores Inteligentes. Informática na Educação: teoria \& prática, Porto Alegre, v. 6 , n. 2, p. 21-37, jul./dez. 2003. 


\section{Contexto da computação afetiva}

Ao longo do sÈculo $X X$, observou-se o aprofundamento dr-stico do modelo de fragmentaÁ,,o do conhecimento, com vÈrtices na ciĺncia, na educaÁ,,o e na produÁ,,o industrial tecnolÛgica. Entretanto, na dÈcada de 1990, retomou-se a discuss,,o de uma nova epistemologia que supere o modelo puramente racionalista, dualista e fragmentador em vigíncia (PRIGOGINE, 1996; MORIN, 1996).

Fortemente impregnadas pelo pensamento racionalista cartesiano, as ciĺncias modernas entendem a emoÁ,,o como algo totalmente a parte da raz,,o (do raciocìnio consciente). Entretanto, esse conceito passou a sofrer mudanÁas significativas quase que paralelamente ao surgimento das chamadas ciĺncias cognitivas, embora inicialmente se admitisse o mecanismo emocional ainda que apenas como uma espècie de ìgerador de opÁıesî a serem decididas/ selecionadas por outro mecanismo, puramente racional e independente do primeiro. Resultados recentemente obtidos, principalmente no campo da neurociĺncia moderna (computacional), apontam uma forte ligaÁ,,O das emoÁıes com quase todos os aspectos da cogniÁ,o e com a origem do pensamento consciente na crianÁa (DAM $\mathrm{SIO}, 1996)$. A noÁ,o de cogniÁ,o inclusive passa a integrar tambÈm a emoÁ,,o e afetividade para alguns pesquisadores (ORTONY, CLORE e COLLINS, 1999). Esse novo entendimento das relaÁıes entre emoÁ,,o, cogniÁ,,o e consciĺncia comeÁou a influenciar, sobretudo na 'Itima dĖcada, alguns projetos de sistemas computacionais em geral, assim como a pesquisa e o desenvolvimento de ambientes de aprendizagem baseados no computador em particular.
As iniciativas da computação afetiva s,,o exemplos de um passo nessa direÁ,,o. Considere-se um significativo conjunto de tĖcnicas de computaÁ,,o, adaptadas de ·reas diversas como a InteligÍncia Artificial (IA) e a Engenharia de Software, agregadas de uma maneira menos fragmentada e coordenadas conjuntamente ao estudo, ao modelamento e ¥ simulaÁ,,o da experí́ncia emocional humana, como caracterl̀stica entremeada e constituidora dos processos cognitivos. Todo esse aparato, orientado a aplicaÁıes em domìnios bastante variados, passou a ser disting sido sob a denominaÁ,,o genÈrica (e ainda n,o plenamente sedimentada) de computaÁ,,o afetiva (PICARD, 1997). A grosso modo pode-se fazer uma distinÁ,,O entre dois grandes grupos de sistemas relacionados ¥ computaÁ „o afetiva:

a) os que consideram a emoÁ,o do usu-rio; por exemplo, um ambiente computacional de ensino-aprendizagem que busca inferir, avaliar e tomar decisıes sobre o estado cognitivo-afetivo do aprendente, a partir da observaÁ,,o da interaÁ,,o deste, ou por outro meio de monitoramento (sensores fisiolûgicos, por exemplo);

b) os que consideram a emoÁ,,o do prÛprio sistema computacional (emoÁ,,o de m.quina); por exemplo, atravÈs da simulaÁ,,o de estados emocionais com o objetivo de regular as aÁıes, a tomada de decisıes e a aprendizagem de um sistema sobre o seu domìnio de aplicaÁ,,o (aprendizagem de m·quina).

Este trabalho apresenta e discute a introduÁ,,o de caracterl̀sticas afetivas no projeto de sistemas que utilizam a tecnologia de agentes ou de multiagentes (SMA) e Sistemas Tutores Inteligentes (STI). Faz-seessa distinÁ,,O, porque embora um STI possa ser 
projetado e implementado atravÈs da tÈcnica de SMA, isto $\mathrm{n}, \mathrm{o}$ È necessariamente uma regra. AlĖm disso, h. modelos interessantes de SMA cognitivo-afetivos em outros domìnios de aplicaÁ,,o que n,,o o do processo ensino-aprendizagem. Como metodologia expositiva ser,, 0 apresentadas iniciativas e abordagens de alguns grupos de pesquisa que consideram a inclus, ,O da emoÁ,,o em SMA e/ou em STI, bem como alguns dos aspectos da fundamentaÁ,,o e da implementaÁ,,o pr.tica (aspectos tecnolÛgicos) desses sistemas.

\section{Computação afetiva na Univer- sidade de Memphis}

Partindo de uma abordagem representacionista, o Grupo de Pesquisa sobre Software Consciente do Laboratório de Ciência Cognitiva ${ }^{1}$ da Universidade de Memphis trabalha no estudo de representaÁıes da inteligíncia e da consciĺncia humanas e na construÁ,,O de artefatos computacionais (notadamente SMA) que expressem tais modelos. Bogner, Ramamurthy e Franklin (1999) apresentam o protÛtipo CMattie ${ }^{2}$ (ìConscious" Mattie) como um agente de software ìconsciente $\hat{i}^{3}$, capaz de interagir, adaptar-se e aprender num ambiente social com agentes de seu tipo e/ou agentes humanos. CMattie possui um mecanismo emocional que influencia seu comportamento, sobretudo via regulaÁ,o de seus drives. ${ }^{4}$

\subsection{O Projeto CMattie}

O projeto CMattie est $\cdot$ fundamenta- do na teoria psicolÛgica da consciĺncia conhecida como EspaÁo de Trabalho Global ${ }^{5}$ (ETG), de acordo com a qual a cogniÁ,,o humana È formada por um grande $n$ ' mero de pequenos processos de propÛsitos especiais e individualmente inconscientes, a partir do pressuposto que o sistema nervoso È um sistema paralelo e distribuldo com muitas unidades processadoras diferentes e especializadas (BAARS, 1988, 1997). Do ponto de vista pr.tico, CMattie Ė a geraÁ,,o seguinte do projeto Virtual Mattie que Ė um SMA n,o ìconscienteî (FRANKLIN et al., 1996). Suas tarefas essenciais consistem em receber, filtrar, responder, reescrever e enviar, atravĖs de mensagens de e-mail escritas em linguagem natural, a divulgaÁ,,o de eventos cientìficos de um departamento, tais como semin.rios, workshops e defesas de tese. CMattie iviveî em um sistema computacional real baseado em UNIX, e suas unidades processadoras $\mathrm{b} \cdot$ sicas $\mathrm{s}, \mathrm{O}$ denominadas codelets.

\subsubsection{Arquitetura emocional de CMattie}

O projeto CMattie apresenta uma complexa estrutura modular, ilustrada na Figura 1. Cada mÛdulo/mecanismo implementa um modelo/teoria de IA especìfica, conforme apresentado na Tabela 1. A implementaÁ,,o do ETG na arquitetura de CMattie È sustentada principalmente na Teoria do PandemÙnio, inicialmente descrita (e aplicada apenas $\ddagger$ percepÁ,,o) por Selfridge (1959) e posteriormente estendida a outros aspectos por Jackson (1987).

\footnotetext{
1 http://mnemosyne.csl.psyc.memphis.edu

2 http://www.msci.memphis.edu/ cmattie

$3 \mathrm{Na}$ concepÁ,,o expressa por Bogner, Ramamurthy e Franklin (1999), um agente de software ìconscienteî È uma entidade que atua em um domìnio especilfico e que implementa o ETG de Baars $(1988,1997)$. Os termos ìconsciĺnciaî e ìconscienteî foram mantidos entre aspas ao longo do texto, seguindo a mesma notaÁ,,o reducionista dos autores. $4 \mathrm{O}$ termo drive pode ser entendido no contexto como vontade, desejo, um tipo de direcionamento das aÁıs. 5 TraduÁ,o livre de Global Workspace, tambĖm citado na literatura como EspaÁo Operacional Global.
} 
Essa arquitetura global ser. abordada muito resumidamente, porque, neste trabalho, o mais importante $\mathrm{E}$ a arquitetura do mecanismo emocional de CMattie, descrita detalhadamente por McCauley e Franklin (1998).

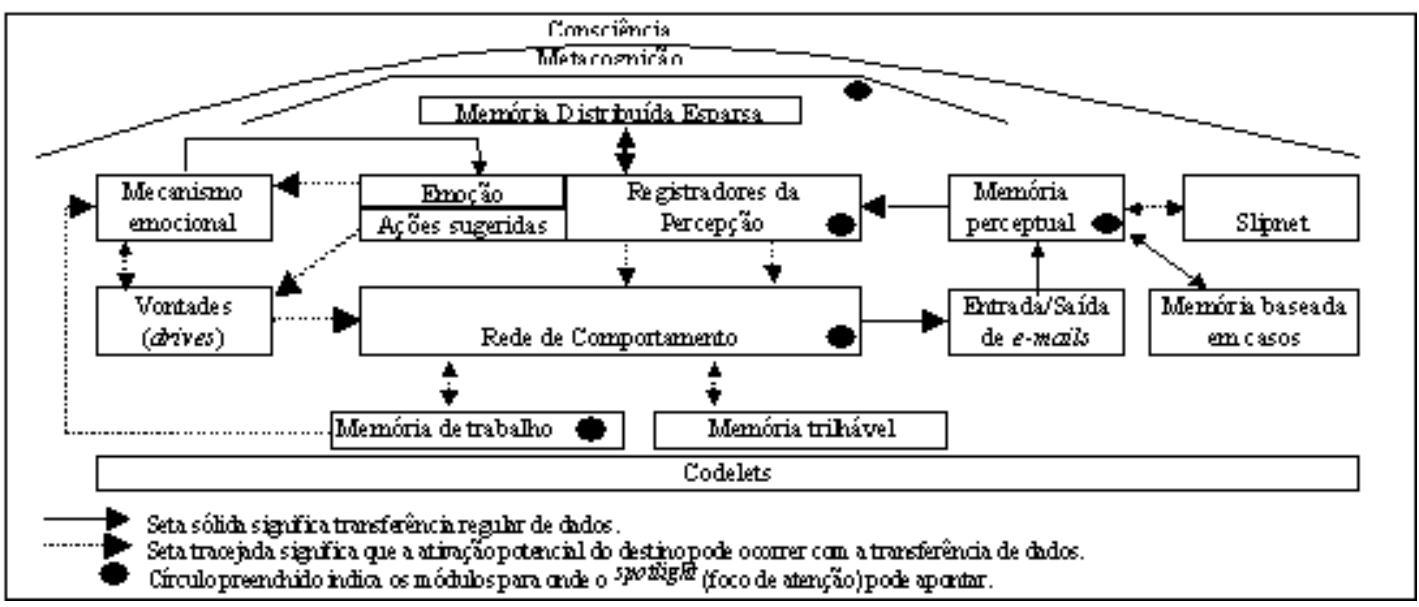

Figura 1 ñ Arquitetura do SMA Cmattie (adaptado de BOGNER, RAMAMURTHY e FRANKLIN, 1999, p. 117).

Tabela 1 ñ MÛdulos e mecanismos de CMattie e teorias/modelos de IA associados.

\begin{tabular}{|c|c|}
\hline Módulo Mecanismo & Modelo de lA adotado \\
\hline Percepção & Por an alogia (MilTCHEШ, 1993) \\
\hline Compreersão dos e-mails & Entendimento de Linguagem Natur al (ALLEN, 1995) \\
\hline Seleção de açốes & Rede de Comportamento (MAES, 1990) \\
\hline Memória associativa & Memória Distribuída Esparsa (KANERVA, 1988) \\
\hline Memória episódica & Memória Baseada em Casos (KOLODNER, 1993) \\
\hline Emoçốes & Teoria do Pandemônio (SELFIDGE, 1959; JACKSON, 1987) \\
\hline Metacognição & Classificador Difuso (HOLLAND, 1986) \\
\hline \multirow[t]{2}{*}{ Aprendizagem } & As quitetura Copycat(HOF STAD TER \& MITCHEШ, 1994), e \\
\hline & Raciocínio Baseado em Cas 0 (KOLODNER, 1993) \\
\hline Geraçăo de linguagem & Teoria do Pandemônio \\
\hline D eliberação & Teoria do Pandemônio \\
\hline "Consciência" & Teoria do Pandemônio \\
\hline
\end{tabular}


A arquitetura de CMattie apresenta diversos drives construldos explicitamente no SMA, que operam em paralelo. Cada drive ativa os fluxos de comportamento que o satisfazem. Alguns exemplos de drives $\mathrm{s}, 0$ : entender cada mensagem recebida; agradecer cada mensagem recebida; manter informaÁ,,o completa sobre cada semin -rio em andamento; manter a lista de e-mails corrente. Em CMattie, a emoÁ,o tem dois papÈis principais: a) afeta a intensidade dos diferentes drives e ,por conseguinte, os nìveis de ativaÁ,,o dos fluxos de comportamento ligados a estes drives; b) influencia as aÁıes sugeridas pela MemÛria distribulda esparsa (Figura 1).

A emoÁ,,o global de CMattie È representada por um vetor de ganho emocional, composto de quatro $n$ 'meros reais an-logos ¥s quatro emoÁıes b-sicas descritas por Izard (1993): raiva, tristeza, felicidade e medo. Existem codelets emocionais din, micos cujas aÁıes resultam na atualizaÁ,o dos valores do ganho da emoÁ,,o correspondente. Este processo consiste de duas etapas. Primeiro s,,o incluldas valíncia, saturaÁ,,o e repetiÁ,,o na intensidade emocional do codelet din,mico, ajustada pela fûrmula:

$$
\begin{gathered}
a=v /\left(1+e^{(-v x+x 0) / 1.5}\right) \\
\text { onde }
\end{gathered}
$$

$\mathrm{a}=$ intensidade emocional do codelet din,mico;

$x=$ intensidade inicial da emoÁ,,o; $v=$ valíncia $\{1,-1\}$; $x_{0}=$ desloca a funÁ,,o $\ddagger$ direita ou $\ddagger$ esquerda.

No segundo passo, cada codelet ativado deve criar uma inst, ncia ìest-ticaî de si mesmo com a intensidade emocional ajustada, que ser-adicionada $\neq$ intensidade global de uma determinada emoÁ,,o atravÈs da fÛrmula (adaptado de PICARD, 1997):

$$
\begin{gathered}
y=a e^{-b(t-t 0)} \\
\text { onde }
\end{gathered}
$$

$y=$ intensidade global de uma determina-

$$
\text { da emoÁ,„o; }
$$

$a=$ intensidade emocional ajustada no momento da criaÁ,,o do codelet; $b$ = taxa de declìnio da emoÁ,,o; $t$ = hora atual; $t_{o}=$ hora da criaÁ,,o do codelet .

\subsection{O Projeto Intelligent Distribution Agent (IDA)}

CMattie representou uma evoluÁ,,o, em termos de arquitetura de SMA, em relaÁ,o ao projeto VMattie. Entretanto, CMattie vem sendo sucedido pelo projeto IDA, ${ }^{6}$ um SMA que apresenta uma arquitetura emocional de vanguarda (MCCAULEY, FRANKLIN e BOGNER, 2000). A Tabela 2 traz um resumo das principais caracterl̀sticas mantidas entre os projetos CMattie e IDA, dentre as quais cabe ressaltar a arquitetura geral baseada no ETG, a forma de comunicaÁ,o com o meio ambiente, o princlipio de aprendizado $e$ algumas caracterl̀sticas ligadas $\ddagger$ emoÁ,,o.

Influenciado pelo entendimento mais recente do mecanismo emocional humano, o projeto IDA incorpora a emoÁ,,o como uma caracterìstica pervasiva da inteligíncia e, portanto, aderente e posicionada nos entremeios de todos os mÛdulos cognitivos do SMA. A Tabela 3 apresenta as principais caracterl̀sticas de evoluÁ,,o de CMattie para

6 http://www.msci.memphis.edu/ ida 
Tabela 2 ñ Principais caracterl̀sticas que foram mantidas entre CMattie e IDA.

\begin{tabular}{|c|c|}
\hline $\begin{array}{l}\text { Característica } \\
\text { Arquitetura geral } \\
\text { Comunicaçẩo } \\
\text { externa }\end{array}$ & $\begin{array}{l}\text { Des crição } \\
\text { Fundamentada na Teoria do ETG (BAARS, 1988, 1997). } \\
\text { Via emails em linguagem natura! }\end{array}$ \\
\hline $\begin{array}{l}\text { Mecanismo de } \\
\text { aprendizado }\end{array}$ & $\begin{array}{l}\text { Adota o principio hebbiano, por reforço baseado na } \\
\text { repetiça e proximidade temporal dos eventos (HEBB, } \\
\text { 1949). }\end{array}$ \\
\hline $\begin{array}{l}\text { Aprendizado } \\
\text { emocional }\end{array}$ & $\begin{array}{l}\text { Possibinta aprender sobre quais tipos de situações geram } \\
\text { guais estadosemocionais. }\end{array}$ \\
\hline $\begin{array}{l}\text { Origem das } \\
\text { emoçốes }\end{array}$ & $\begin{array}{l}\text { Ocorrem em resposta a estímulas externas e estados } \\
\text { internos. }\end{array}$ \\
\hline Emoçốes básicas & $\begin{array}{l}\text { Assumem uma representação explicita, em um vetor } \\
\text { dinamico de quatro (CMattie) ou seis (IDA) vabres reais. }\end{array}$ \\
\hline $\begin{array}{l}\text { Estados emocionais } \\
\text { complexos }\end{array}$ & $\begin{array}{l}\text { Assumem uma representação implicta, emergente da } \\
\text { combinação dinâmica dos valores das emocoues básicas }\end{array}$ \\
\hline
\end{tabular}

Tabela 3 ñ Caracterl̀sticas que foram modificadas e/ou estendidas de CMattie para IDA.

\begin{tabular}{|c|c|c|}
\hline Característica & CMattie & IDA \\
\hline Domínio'Tarefa & $\begin{array}{l}\text { Preparar e ewiar anuncios } \\
\text { semanais de eventos } \\
\text { cientificas na Universidade } \\
\text { de Memphis. }\end{array}$ & $\begin{array}{l}\text { Trabaihar como "detailer" para a } \\
\text { marinha dos EUA, ou seja, } \\
\text { negociar a distribuiça e o } \\
\text { agendamento das atividades dos } \\
\text { marinheiros. }\end{array}$ \\
\hline $\begin{array}{l}\text { Seleçẫo de } \\
\text { açốes }\end{array}$ & $\begin{array}{l}\text { Emog öes afetam a escoha } \\
\text { de determinado } \\
\text { comportamento e avaliam } \\
\text { os resultados dessas aços, } \\
\text { gerando aprendizado. }\end{array}$ & $\begin{array}{l}\text { Deve ser capaz de deliberar sobre } \\
\text { vários cenários (planejamento) e } \\
\text { negociar com humanos } \\
\text { (estratégia). }\end{array}$ \\
\hline $\begin{array}{l}\text { Influência da } \\
\text { emoçẫo no } \\
\text { comportamento }\end{array}$ & $\begin{array}{l}\text { Emoção afeta o } \\
\text { comportamento } \\
\text { indiretamente, reforçando } \\
\text { ou enfraquecendo os dives } \\
\text { que regulam as fiuxos de } \\
\text { comportamento. }\end{array}$ & $\begin{array}{l}\text { Emoça integrada no mecanismo } \\
\text { da "consciência" e conectada } \\
\text { bidiecionaimente às demais } \\
\text { partes da arquitetura, funcionando } \\
\text { como uma "moeda de troca" entre } \\
\text { elas. }\end{array}$ \\
\hline $\begin{array}{l}\text { Emoçỗes } \\
\text { básicas }\end{array}$ & $\begin{array}{l}\text { Rava, tristeza, felicidade e } \\
\text { medo. }\end{array}$ & $\begin{array}{l}\text { Além das quatro foram } \\
\text { acrescentadas desgosto e } \\
\text { surpresa. }\end{array}$ \\
\hline
\end{tabular}

IDA. Um fator determinante na adoÁ,,O do novo modelo de emoÁ,,o advÈm de um domìnio de aplicaÁ,o mais amplo que o anterior: fazer o papel de um ìdetailerî para a marinha norte-americana. Tal domìnio exige aÁıes de planejamento para diversos cen-rios possìveis e a adoÁ,,o de estratĖgias de negociaÁ,,o com hu- manos para atribuir missıes aos marinheiros, respeitando as restriÁıes de datas, hor-rios, missıes, famìlia, legislaÁ,,o e situaÁıes de exceÁ,,o. A complexidade das tarefas nesse domìnio tambÈm justifica o modelamento explìcito das emoÁıes desgosto e surpresa, n,o adotadas em CMattie. 


\subsection{Computação afetiva e a IA rígida}

Os projetos do Grupo de Memphis aqui relatados representam uma das principais iniciativas do primeiro tipo de abordagem de computaÁ,,o afetiva: a incorporação da emoção no projeto de sistemas computacionais baseados em SMA cognitivos. A pretens,,O de modelar representaÁıes da cogniÁ,,o, aprendizagem, emoÁ„,o e ìconsciĺnciaî sobre um artefato computacional (m.quina, algoritmo, SMA), ainda que em car-ter assumidamente reducionista, acende (ou reacende) a discuss,,o dos fundamentos sobre os limites da IA em relaÁ,o abordagem representacionista È condizente com a chamada IA rl̀gida, a qual sustenta que qualquer processo mental $\boldsymbol{A}$ nada mais È se$\mathrm{n}$, o um processo $\boldsymbol{B}$ de IA (HORGAN, 1996, apud $\mathrm{KOV}_{i} \mathrm{CS}$, 1997) e recebe crìticas de pesquisadores que buscam argumentos fenomenolÛgicos: o pensamento não seria formalizável(DREYFUS, 1972, 1992); matem.ticos e lÛgicos: em geral baseados no Teorema da Incompletude de Gödel por exemplo Kov.cs (1997); anti-behavioristas: uma máquina (algoritmo) operaria no domínio sintático e nunca no domínio semântico (SEARLE,1980); alĖm dosargumentos Èticos: a essência do humano não poderia ser reduzida ao paradigma do processamento de informações do Modelo Computacional da Mente. ${ }^{8}$ Colocando-se em outro domìnio de observaÁ,,o, ‡ parte dos fundamentos da Fi- losofia da Mente e dos argumentos favor-veis ou contr.rios $¥ \mathrm{IA}$ rìgida, a adoÁ,,o dessa abordagem mostrar resultados pr-ticos promissores. $^{9}$

Dam·sio (1996) propıe a evoluÁ,,o da consciĺncia humana ao longo da histÛria filogenÈtica da espÈcie a partir de um tipo de emoÁ,,o ancestral. De certa forma, esses projetos de SMA cognitivo-afetivos acompanham as mudanÁas no conceito da relaÁ,,o entre emoÁ,,o, cogniÁ,,o e consciĺncia. $\mathrm{Na}$ sucess, ,o de VMattie para CMattie houve a incorporaÁ,,o da emoÁ,,o, com efeitos indiretos sobre a tomada de decisies e outros aspectos cognitivos do SMA, atravÈs da regulaÁ,,o de seus drives. Dito de outra forma, em CMattie o mecanismo da emoÁ,,o est relativamente isolado em um mûdulo, que tem papel importante, mas secund.rio. A evoluÁ,,O de CMattie para IDA ocorreu justamente pela ampliaÁ,,o do papel da emoÁ,o, que passa a entremear todas as funÁıes cognitivas (por exemplo os v-rios tipos de memÛria e o processo de tomada de decis,,o) e, por conseguinte, todo o comportamento do agente. Soma-se a isso a ampliaÁ,o do espectro de emoÁres b-sicas como estratĖgia para lidar com domìnios de maior complexidade. Em resumo, no agente IDA, os mÛdulos cognitivos se comunicam, trocando mensagens de conte 'do conceitual e afetivo.

7 O matem·tico Kurt G^del demonstrou que nenhum sistema formal consistente È completo. Isso delimita a capacidade computacional de uma m.quina algorltmica (NAGEL e NEWMAN, 1973, apud KOV ${ }_{i}$ CS, 1997). Em tempo, os limites do complexo mente/cĖrebro, embora aceitos, ainda n„o foram demonstrados

8 Resumidamente, segundo o paradigma do Modelo Computacional da Mente, o cĖrebro funciona como um processador de informaÁıes e a mente seria uma espècie de software que ìrodaî no hardware do cĖrebro (sistema nervoso). 9 Os resultados de desempenho apresentados, tais como a precis,,o na geraÁ,,o e envio de an “ncios de eventos, s,,O relativos ao projeto VMattie. O projeto CMattie fùra implementado em cerca de $40 \%$ atÈ a publicaÁ,o de Bogner, Ramamurthy e Franklin (1999). O projeto IDA est· em fase de implantaÁ,o e, atÈ o momento, n„o h. resultados publicados. 


\section{Computação afetiva no Instituto Tecnológico de Massachussets (MIT)}

Liderado por Rosalind Picard, o Grupo de Pesquisa em Computação Afetiva ${ }^{10}$ do Laboratório de Mídia ${ }^{11}$ do MIT È o principal exemplo da adoÁ,,o do conceito moderno da relaÁ,,o entre cogniÁ,,o e emoÁ,,o no projeto de sistemas inteligentes. Entre suas linhas de investigaÁ,,o est,,o a pesquisa sobre emoÁıes humanas, sensoriamento de sinais afetivos humanos, reconhecimento de padries de expressies afetivas, modelamento da experiĺncia emocional, sintetizaÁ,,o de emoÁıes em m·quinas, aplicaÁıes de computaÁ,o afetiva, interfaces com computadores afetivos, comunicaÁ,,o afetiva e computadores afetivos utiliz-veis. Observa-se que o escopo de atuaÁ,,o do MIT È bastante mais extenso do que a ìsimplesî incorporaÁ,,o de emoÁ,,o a SMA ou a STI, embora isso ocorra nas aplicaÁıes de computaÁ,,o afetiva.

\subsection{O projeto Learning Companion}

Um projeto em desenvolvimento no Grupo de Pesquisa em Computação Afetiva do MIT, interessante sobretudo para aplicaÁ,,o em STI, refere-se a um ìassistente de aprendizagemî (Learning Companion). Proposto por Kort, Reilly e Picard (2001), este projeto trata do modelamento de emoÁıes possivelmente relevantes para aprendizagem e da posterior construÁ,,o de um artefato computacional capaz de reagir adequadamente ao estado afetivo e cognitivo do aluno, atravÈs do ajuste imediato da estratÈgia de ensino. O projeto do assistente fundamenta-se na idĖia de que diferentes emoÁres ou suas respostas conscientes, os sentimentos, $\mathrm{s}$, o naturais ao processo de apren- dizagem. Por exemplo, no desenvolvimento da ciĺncia ou da tecnologia, os cientistas s, O submetidos ao erro e $¥$ frustraÁ,,o como parte do seu processo de aprendizado. Esses nìveis de falha s,,o parte do processo e podem ensinar como aprender melhor.

\subsubsection{Arquitetura de emoção do Learning Companion}

... importante ressaltar a preocupaÁ,,o deste projeto com o modelamento do estado afetivo-cognitivo do aprendiz. Oassistente n, o necessariamente deve possuir ou expressar emoÁıes, mas ser capaz de reagir satisfatoriamente $\ddagger$ emoÁ,,o do aluno. O conjunto de emoÁıes ìcandidatas a relevantesî para a aprendizagem, em sua vers,,o atualizada, descrita por Kort e Reilly (2002), È composto de seis eixos afetivos que apresentam graduaÁıes vari-veis entre os pares de extremos mutuamente exclusivos: da ansiedade $\ddagger$ confianÁa, da chateaÁ,,o ‡ fascinaÁ,,o, da frustraÁ,,o $\ddagger$ euforia, do des,nimo ao encorajamento, do terror ao encantamento, da humilhaÁ,o ao orgulho. Acredita-se que a primeira parte de cada par seja um afeto de valíncia negativa e a segunda parte, um afeto de valíncia positiva para o aprendizado. A representaÁ,,o proposta para correlaÁ,,o entre o estado afetivo e o estado cognitivo do aprendiz est. ilustrada na Figura 2. O conhecimento emergiria em um plano ortogonal ao plano dos eixos de aprendizagem e afeto, e sua construÁ,,o pelo aluno se daria, ao longo do tempo, de maneira cl̀clica e elicoidal, passando por fases afetivo-cognitivas positivas e negativas.

A validaÁ,o empìrica do modelo È feita atravÈs da filmagem de crianÁas de 6 


\section{teoria \& prática}

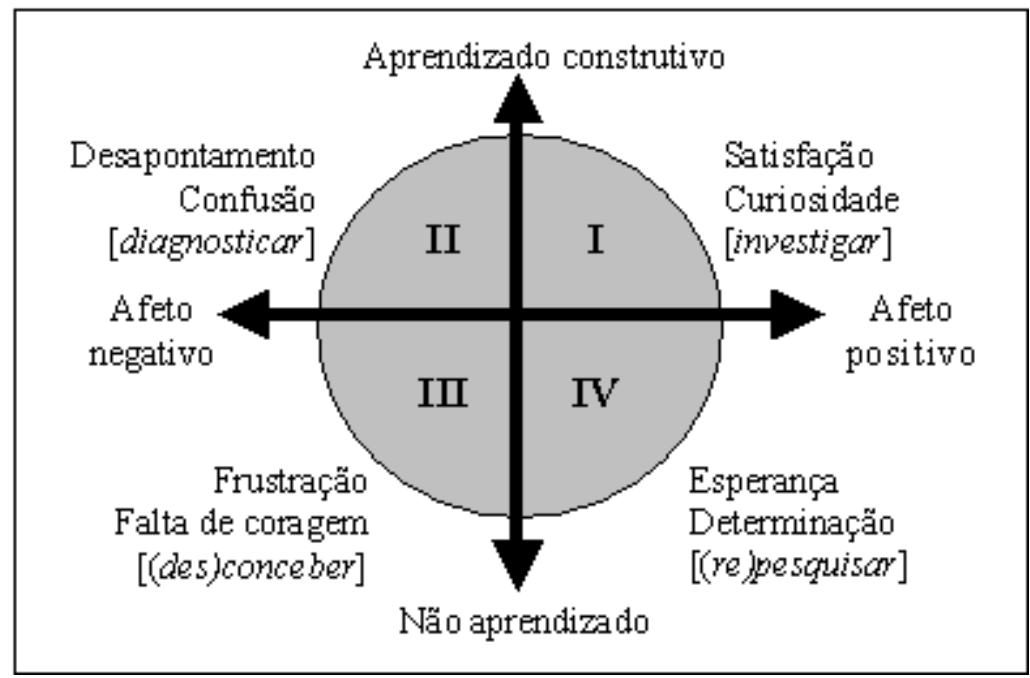

Figura 2 ñ Modelo de eixos de relacionamento entre aprendizagem e emoÁ,o no Learning Companione as aÁıes mais representativas associadas a cada estado cognitivo-afetivo do aluno (adaptado de KORT e REILLY, 2002).

a 11 anos de idade, enquanto estas utilizam alguns jogos de computador. Uma c,mera segue o movimento dos olhos da crianÁa para determinar para onde ela est. olhando em cada momento, enquanto outras c,meras capturam suas expressies faciais, posturais e gestuais. Os dados obtidos s,,o utilizados para ajustar o modelo cognitivo-afetivo do aluno, atravÈs da correlaÁ,,o entre o estado emocional suposto, o comportamento ao nìvel de superfìcie e o estado ou tarefa do jogo no mesmo momento. Com base nesses resultados, pretende-se construir um artefato computacional capaz de reagir ajustando a estratĖgia de ensino de acordo com o estado afetivo-cognitivo do aluno. AtÈ o momento n,,o s, o conhecidos resultados publicados alÈm desta etapa.

\section{Computação afetiva na Univer- sidade Federal do Rio Grande do Sul (UFRGS)}

O Grupo de Pesquisa em Inteligência Artificial (GPIA) do Instituto de Informática da UFRGS tem se dedicado, entre outros projetos, ‡ pesquisa e ao desenvolvimento de agentes pedagûgicos inteligentes para aplicaÁ,,o em STI, por exemplo nos trabaIhos de Moussale (1996), Giraffa (1999), Bercht (2001), Silveira (2001), Bocca (2003) e Jaques et al. (2003). Destaca-se o desenvolvimento recente de SMA cognitivos segundo a abordagem de estados mentais da arquitetura BDI, ${ }^{12}$ por exemplo em Giraffa (1999) e Bercht (2001), atravÈs de um ambiente-base para construÁ,o de agentes cognitivos, denominado X-BDI, um modelo formal e execut-vel descrito por MÛra (2000). 
A noÁ,,o de agente pedagûgico vai designar alguns dos diferentes sistemas aplicados ‡ educaÁ, ,o desenvolvidos com uso da tecnologia de agentes. O objetivo È auxiliar os alunos no processo de aprendizagem. Esses agentes podem ser modelados como (1) agentes que cooperam trabaIhando na retaguarda como parte da arquitetura de um sistema educacional ou (2) agentes animados que interagem com o usu-rio atravÈs de um personagem. Por exemplo, pode-se adotar a figura de um personagem ìamigoî ou de um personagem de um ìcriador de problemasî, instigando e/ou dando dicas das tarefas a serem realizadas. Neste caso, tem-se o agente pedagÛ́gico como um elemento de um STI e as pesquisas do GPIA/UFRGS levaram ao desenvolvimento da personagem PAT (BOCCA, 2003), conforme ilustrado na Figura 3. Outra forma È a adoÁ,,o do agente pedag Ûgico atuando em todo o comportamento e interaÁ,,o do ambiente ou STI, sem aparecer ao aluno explicitamente como um personagem, conforme adotado no projeto de Bercht (2001).

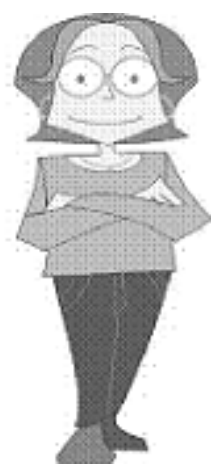

Figura 3 ñ Personagem PAT: representaÁ,,o do agente pedagÛgico animado descrito por Bocca (2003).

Dal̀ a necessidade de trazer o raciocl̀nio sobre afetividade ao projeto de agentes pedagûgicos. De modo a haver sintonia e credibilidade, esses agentes pedagûgicos atores devem responder com faculdades afetivas, tal como atravÈs de expressıes faciais que reflitam o estado emocional necess-rio ‡ ocasi,,o. Mas, o que se est. advogando È uma preocupaÁ,,o em pesquisas sobre as necessidades dos agentes pedagÛgicos em melhorar suas atuaÁıes (flexibilizaÁıes de suas estratĖgias), de maneira a impactar positivamente o modo do aluno perceber a situaÁ,,o de aprendizagem. Uma das propostas È a inserÁ,,o da afetividade envolvida na situaÁ,,o como parte do raciocìnio das aÁıes que o agente deve conduzir.

\subsection{Agentes pedagógicos com dimen- sões afetivas}

Bercht (2001) descreve uma arquitetura de STI, que apresenta um mÛdulo de percepÁ,,o composto por agentes especializados integrados com a interface do tutor, para rastrear a interaÁ,,o do aluno durante a tutoria. Os dados coletados pela PercepÁ,o permitem compor um histÛrico e fazer inferíncias sobre o comportamento do 
aluno, tanto no que concerne ao seu desempenho (relativo ao domìnio) quanto aos seus aspectos afetivos e motivacionais. $O$ modelo adotado È composto de duas dimensıes: Esquemas Intelectuais e Esquemas Afetivos (Figura 4), gerados por inferíncia do tutor (aÁ,,o do ìkernel cognitivoî) sobre as caracterìsticas (observ-veis) surgidas durante a interaÁ,,o. Por exemplo, um dos agentes da PercepÁ,,o projetado por Bercht (2001) indentifica fatores motivacionais do aluno, tais como a persistíncia (esforÁo), a independíncia e a confianÁa demonstradas durante a interaÁ,,o. Estes esquemas $\mathrm{s}$, o representados como estados mentais do aluno, como um conjunto de CrenÁas no ambiente de desenvolvimento X-BDI.

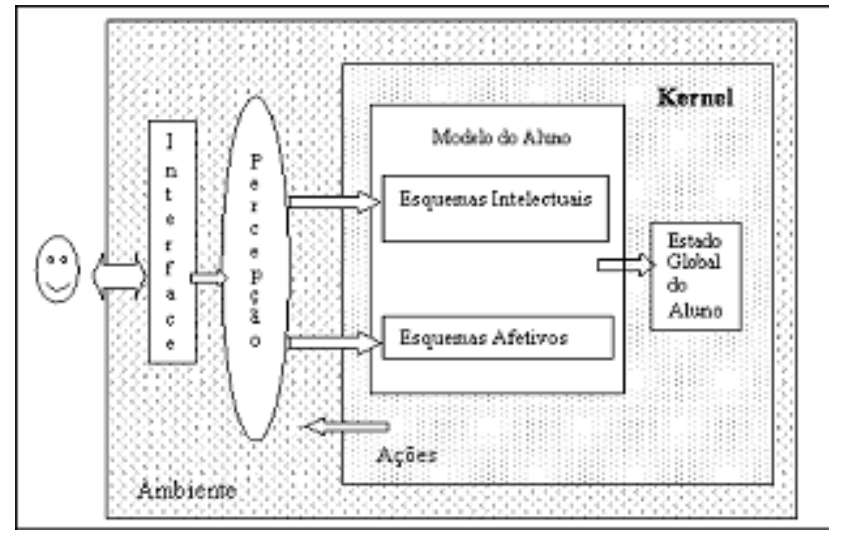

Figura 4 ñ Modelo do aluno com fatores intelectuais e afetivos integrados (reproduzido do original de BERCHT, 2001).

O modelo do aluno È implementado como crenÁas do tutor sobre o estudante, armazenadas no mÛdulo de representaÁ,,o interna de cada aluno em interaÁ,,o. O esquema intelectual contÈm as informaÁıes sobre as condiÁıes do desempenho do aluno na execuÁ,,o de tarefas e o outro re-
tÈm as informaÁıes sobre as caracterìsticas afetivas. Um exemplo da modelagem de um fator motivacional considerado $\tilde{n}$ confiança ñ È descrito nas regras da Tabela 4. A confianÁa do aluno pode ser representada como um valor que varia entre $-2 \mathrm{e}+2$.

Tabela 4 ñ Modelo do nl̀vel de confianÁa do aluno, segundo as regras adotadas por Bercht (2001).

\begin{tabular}{|l|l|c|}
\hline \multicolumn{1}{|c|}{ Situação } & Estado de Suporte & Confiança \\
\hline No tentative & Ask assistance & -1 \\
\hline Succeed & With assistance & +1 \\
\hline Succeed & Without assistance & +2 \\
\hline Failure & Without assistance & -1 \\
\hline Failure & With assistance & -2 \\
\hline
\end{tabular}


Estes trís fatores (esforÁo, confianÁa e independíncia) referem-se ao modelo de motivaÁ,,o aparente e observ -vel do aluno. A motivaÁ,o do aluno se altera durante o processo de interaÁ,,o com o sistema, afetada por diversas causas e determinando a sensaÁ,,o ou emoÁ,,o de ìdesgostarî. $O$ estado afetivo de desgosto pode determinar decrÈscimo da motivaÁ,,O do aluno. Assim, este trabalho realiza uma previs, ,o do estado afetivo do aluno atra-
vĖs da teoria de avaliaÁ,,o cognitiva OCC de Ortony, Clore e Collins (1999).

Esses modelos foram testados atravÈs do Eletrotutor III, uma inst, ncia produzida no JADE Environment (SILVEIRA, 2001). Na Figura 5 est - ilustrada a interface do Eletrotutor. Na parte inferior, $\ddagger$ direita, È possìvel visualizar o grau dos estados afetivos esforÁo (E), confianÁa (C) e independÍncia (I) do estudante, os quais foram detectados pelo agente.

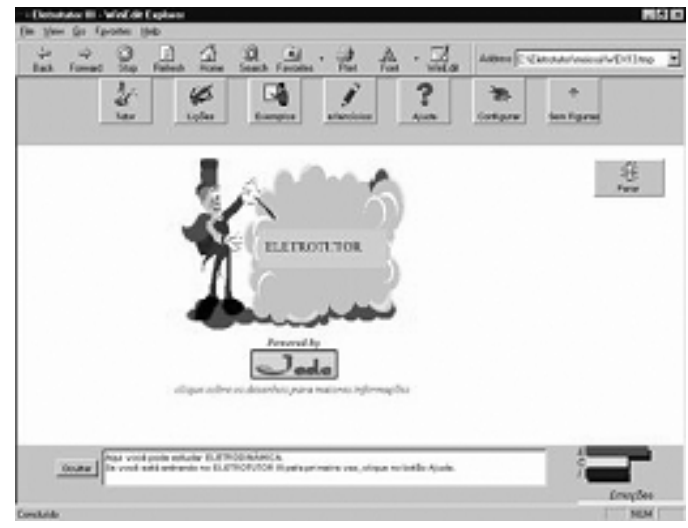

Figura 5 ñ Interface do Eletrotutor III melhorada com a modelagem afetiva do estudante.

\section{Considerações finais}

Os resultados recentes na pesquisa sobre as emoÁıes humanas vím causando mudanÁas no entendimento do papel dos fatores afetivos no processo racional consciente. Aos poucos, essa evoluÁ,,o vem-se refletindo na pesquisa e no desenvolvimento de sistemas computacionais inteligentes, tais como SMA e STI.

Embora tema relativamente recente, $\mathrm{n}$, o se objetiva aqui esgotar as iniciativas que consideram a emoÁ,, SMA e STI. Cabe destacar algumas iniciativas importantes que $\mathrm{n}_{\text {,o }}$ foram detalhadas anteriormente. O projeto AutoTutor ${ }^{13}$ (GRAESSER et al., 2001) propıe um agente pedagÛ́gico que utiliza a estratÈgia da conversaÁ,,o e, para tanto, apresenta emoÁıes e procura interpretar o estado emocional do aluno atravĖs de di -logos escritos. $O$ STI, descrito como um agente credìvel por Abou-Jaoude e Frasson (1998), em adapta- 
Á,o do modelo proposto por Elliott (1997), apresenta um conjunto de emoÁıes b.sicas. E o projeto de integraÁ, ,O de computaÁ,,O afetiva em agentes tutores liderado por Clark Elliott ${ }^{14}$, James C. Lester ${ }^{15}$ e Jeff Rickel ${ }^{16}$. Certamente tambÈm mereceriam espaÁo, em um levantamento mais minucioso, tanto o raciocinador afetivo de Elliott (1992) e sua vers,, o adaptada para STI (1997), quanto o sistema Cathexis (VELiSQUEZ, 1997), que modela a emoÁ,,o em agentes atravĖs de tÈcnicas de redes neurais.

Observa-se, na maioria dos trabalhos de computaÁ,,O afetiva sobre SMA e/ou STI, a adoÁ "o de emoÁres b.sicas conforme os conjuntos propostos por Ekman (1992), Izard (1993) e Picard (1997), ou suas variaÁıes destes. Entretanto, a forma como essas emoÁıes b-sicas s, , o processadas e interpretadas varia significativamente em funÁ,,O do contexto. Em STI pode ser mais importante modelar, inferir e decidir sobre o estado afetivo do aluno do que necessariamente o sistema possuir ou expressar emoÁıes. Em SMA aplicados a outros domìnios de interaÁ,o, o agente apresentar, possuir e/ou expressar emoÁıs pode ser mais importante do que a capacidade de inferir o estado emocional de quem interage com o sistema.
Os projetos CMattie e IDA do Grupo de Memphis, SMA com um modelo de ìconsciĺnciaî que integra aprendizagem e emo$\hat{A}_{\text {, }}$, , assim como outros modelos inspirados na interconex,,o maciÁa que ocorre no cĖrebro, tendem a apresentar uma sobrecarga no mecanismo de troca de mensagens, o que geralmente se reflete em um custo computacional alto.

Destaca-se, no trabalho de Bercht (2001) do PGIA/UFRGS, o uso de agentes especializados que permitem a modelagem do aluno a partir de observ veis durante a interaÁ,,o, atravĖs de uma representaÁ,,o de alto nìvel que integra os fatores cognitivos e afetivos do aluno, na forma de CrenÁas em X-BDI.

Diferentemente, a lÛgica que permeia o Projeto Learning Companion do MIT È a validaÁ,,o empìrica a prioride estratĖgias de ensino correlacionadas com o estado afetivocognitivo do aluno. Estado este inferido a partir de observ-veis do comportamento global do aluno. A aplicaÁ, ,o de tal modelo exige o monitoramento do aluno atravĖs de c,meras e pressupie a existíncia de um padr,,O genĖrico de correlaÁ,,o afetivocognitiva. Destaca-se a proposiÁ „o de um modelo que assume o papel e a import,ncia de estados emocionais ìpositivosî e ìnegativosî sobre a aprendizagem. 
ReferÍncias

ABOU-JAOUDE, S.; FRASSON, C. Emotion Computing in Competitive Learning Environments. In: PEDAGOGICAL AGENTS, INTERNATIONAL CONFERENCE ON INTELLIGENT TUTORING SYSTEMS, 4., 1998, San Antonio. Proceedings... San Antonio, 1998. P. 33-39.

ALLEN, J. Natural Language Understanding. Redwood City, CA : The Benjamin Cummings Publishing Company, 1995.

BAARS, B. A Cognitive Theory of Consciousness. New York: Cambridge University Press, 1988.

.In the Theater of Consciousness. New York: Oxford University Press, 1997.

BERCHT, M. Em Direção a Agentes Pedagógicos com Dimensões Afetivas. Porto Alegre:Universidade Federal do Rio Grande do Sul , 2001. Tese de Doutorado em Ciĺncia da ComputaÁ,o ñ Instituto de Inform.tica, Universidade Federal do Rio Grande do Sul, 2001.

BOCCA, E. Modelagem e Implementação da Interface para Apresentação de Comportamentos Animados e Emotivos de um Agente Pedagógico Animado. 2003.152 p. DissertaÁ,o (Mestrado em Ciĺncia da ComputaÁ,o) - Instituto de Inform tica, Universidade Federal do Rio Grande do Sul, Porto Alegre, 2003.

BOGNER, M.; RAMAMURTHY, U.; FRANKLIN, S. ìConsciousnessî and Conceptual Learning in a Socially Situated Agent. In: DAUTENHAHN, K. (Ed.). Human Cognition and Social Agent Technology. Amsterdam: John Benjamins Publishing, 1999.

BRATMAN, M. E. Intention, Plans and Practical Reason. Cambridge: Harvard University Press, 1987.

DAMiSIO, A. R. O Erro de Descartes: Emoção, Razão e o Cérebro Humano. S,o Paulo: Companhia das Letras, 1996.

DREYFUS, H. L. What Computers can't do. MIT Press, 1972.

. What Computers still can't do: A critique of artificial reason. MIT Press, 1992.

EKMAN, P. An Argument for Basic Emotions. In: STEIN, N. L.; OATLEY, K. (Eds.) Basic emotions. Hove, UK: Lowrence Erlbaum, 1992.

ELLIOTT, C. The affective Reasoner: a process model of emotions in a multi-agent system. 1992. Tese (Doutorado em EducaÁ,,o) - Institute for The Learning Sciences, Northwestern University, Chicago, 1992.

. Affective Reasoner Personality Models for Automated Tutoring Systems. In: WORLD CONFERENCE ON ARTIFICIAL INTELLIGENCE IN EDUCATION, 8., 1997, Kobe, Japan. ProceedingsÖ Kobe: 1997.

ELLIOTT, C.; RICKEL, J.; LESTER, J. C. Integrating affective computing into animated tutoring agents. In: IJCAI WORKSHOP ON ANIMATED INTERFACE AGENTS: MAKING THEM INTELLIGENT, 1997, Nagoya, Japan. ProceedingsÖ Nagoya, 1997. P. 113-121.

FRANKLIN, S. et al. Virtual Mattie - an Intelligent Clerical Agent. In: AAAI SYMPOSIUM ON EMBODIED COGNITION AND ACTION. 1996, Cambridge, MA. Proceedings... Cambridge, 1996.

GIRAFFA, L.M.M. Uma arquitetura de tutor utilizando estados mentais. 1999. Tese (Doutorado em Ciĺncia da ComputaÁ„,o) ñ Instituto de Inform·tica, Universidade Federal do Rio Grande do Sul, Porto Alegre,1999.

GRAESSER, A. C. et al. Intelligent Tutoring Systems With Conversational Dialogue. Al Magazine, n. 22, p. 39-51, 2001. 
HEBB, D. The Organization of Behavior. New York: Wiley, 1949.

HOFSTADTER, D.; MITCHELL, M. The Copycat Project: A model of mental fluidity and analogy making. In: HOLOYAK, K.;BARDEN, J. Advances in Connectionist and Neural Computation Theory, 2. Norwood, NJ: Ablex, 1994.

HOLLAND, J. H. A Mathematical Framework for Studying Learning in Classifier Systems. In: FARMER, D. et. al. (Eds.) Evolution, Games and Learning: Models for Adaptation in Machine and Nature. Amsterdam: North-Holland, 1986.

HORGAN, J. The End of Science. Addison Wesley, 1996.

IZARD, C. Four Systems for Emotion Activation: cognitive and noncognitive processes. Psychological Review, v. 1, n. 100, p. 68-90, 1993.

JACKSON, J. Idea for a Mind. Siggart Newsletter, n. 101, p. 23-26, 1987.

JAQUES, P. A. et al. Cognitive Reasoning to Respond Affectively to the Student. In: THE IASTED INTERNATIONAL CONFERENCE ON COMPUTERS AND ADVANCED TECHNOLOGY IN EDUCATION, 2003, Rhodes, Greece. Proceedings... Calgary: ACTA Press, 2003. P. 57-62.

KANERVA, P. Sparse Distributed Memory. Cambridge, MA: The MIT Press, 1988.

KOLODNER, J. Case-based Reasoning. San Mateo, CA: Morgan Kaufmann Publishers, 1993.

KORT, B.; REILLY, R. Theories for Deep Change in Affect-sensitive Cognitive Machines: a constructivist model. Educational Technology \& Society, v. 4, n. 5, 2002.

KORT, B.; REILLY, R.; PICARD, R. W. An Affective Model of Interplay Between Emotions and Learning: reengineering educational pedagogy ó building a learning companion. In: OKAMOTO, T. et al. Advanced Learning Technology: issues, achievements and challenges. Los Alamitos, CA: IEEE Computer Society, 2001.

$K_{\mathrm{KOV}} \mathrm{CS}, \mathrm{Z}$. L. $\mathrm{O}$ Cérebro e a sua Mente: uma introdução à neurociência computacional. $\mathrm{S}_{\text {„O }}$ Paulo: EdiÁ,„o AcadÍmica, 1997.

MAES, P. How to do the Right Thing. Cambridge, MA: Connection Science, 1990.

MCCAULEY, L.; FRANKLIN, S. An architecture for emotion. In: AAAI FALL SYMPOSIUM EMOTIONAL AND INTELLIGENT: THE TANGLED KNOT OF COGNITION, 1998, Menlo Park, CA. ProceedingsÖ Menlo Park: AAAI Press, 1998, p. 122-128.

MCCAULEY, L.; FRANKLIN, S.; BOGNER, M. An Emotion-Based ìConsciousî Software Agent Architecture. In: PAIVA, A. Affective Interactions. Lecture Notes on Artificial Intelligence. v. 1814, Berlin: Springer, 2000.

MITCHELL, M. Analogy Making as Perception. Cambridge, MA: MIT Press, 1993.

M"RA, M. Um Modelo de Agente Executável. 2000. Tese (Doutorado em Ciĺncia da ComputaÁ,o) ñ Instituto de Inform tica, Universidade Federal do Rio Grande do Sul, Porto Alegre, 2000.

MORIN, E. Epistemologia da Complexidade. In: SCHNITMAN, D. F. (Org.). Novos Paradigmas, Cultura e Subjetividade. Porto Alegre: Artes MĖdicas, 1996.

MOUSSALE, N. Interações Tutor-aluno Analisadas Através de seus Estados Mentais. 1996.

DissertaÁ,,O (Mestrado em CiÍncia da ComputaÁ,,o) ñ Instituto de Inform·tica, Universidade Federal do Rio Grande do Sul, Porto Alegre, 1996.

NAGEL, E.; NEWMAN, J. Prova de Gödel. S.I.: Editora Perspectiva, 1973. 
ORTONY, A; CLORE, G; COLLINS, A. The Cognitive Structure of Emotions. Cambridge: Cambridge University Press, 1999.

PICARD, R. Affective Computing. Cambridge, MA: The MIT Press, 1997.

PRIGOGINE, I. O Fim da ciĺncia? In: SCHNITMAN, D. F. (Org.). Novos Paradigmas, Cultura e Subjetividade. Porto Alegre: Artes MĖdicas, 1996.

SEARLE, J. Minds, Brains, and Programs. Behavioral and Brain Sciences, n. 3, p. 417-424, 1980.

SELFRIDGE, O. G. Pandemonium: a paradigm for learning. In: SYMPOSIUM ON MECHANIZATION OF THOUGHT PROCESSES, 1959, London. Proceedings... London: National Physics Lab. / HM Stationery Office, 1959, p. 513-526.

SILVEIRA, R. A. Modelagem Orientada a Agentes Aplicada a Ambientes Inteligentes Distribuídos de Ensino: JADE (Java Agent framework for Distance learning Environments). 2001. Tese (Doutorado em Ciĺncia da ComputaÁ,o) ñ Instituto de Inform ·tica, Universidade Federal do Rio Grande do Sul, Porto Alegre, 2000.

VELiSQUEZ, J. Modeling Emotions and Other Motivations in Synthetic Agents. In: NATIONAL CONFERENCE ON ARTIFICIAL INTELLIGENCE, 14., 1997, Menlo Park, CA. Proceedings... Menlo Park: AAAI Press, 1997.

\section{Edilson Pontarolo}

Mestre em Engenharia ElÈtrica e Inform·tica Industrial (CPGEIñCEFET/PR), professor do CEFET/PR Unidade de Pato Branco, professor visitante na Escola TÈcnica da UFRGS.

E-mail: edi@etcom.ufrgs.br

\section{Magda Bercht}

Doutora em Ciĺncia da ComputaÁ,o (CPGCCñUFRGS), professora do Instituto de Inform-tica da UFRGS e da disciplina ìComputaÁ,o Afetivaî do PGIE/UFRGS.

E-mail: bercht@inf.ufrgs.br

\section{Rosa Maria Vicari}

Doutora em Inform tica (Universidade de Coimbra), professora do Instituto de Inform tica da UFRGS e da disciplina ìComputaÁ,,o Afetivaî do PGIE/UFRGS.

E-mail:rosa@inf.ufrgs.br 